$\mathrm{Cu} / \mathrm{Au}$ system, toward the Au. Measurements agree well with Kirkendall's, showing that the technique of joining is unimportant. The Matano Interface agrees with the original interface, showing conservation of lattice sites. The amount of marker shift differs from system to system, for unexplained reasons; the amount of shift is sometimes near the maximum possible; it is large in $\mathrm{Cu} / \mathrm{Zn}$ and $\mathrm{Cu} / \mathrm{NI}$ systems, smaller in others. Dimensional changes seem to be restricted to the direction parallel to diffusion flow. $\mathrm{Cu} / \mathrm{Zn}$ couples exhibit apparent traces of porosity in the diffuston zone (and anomalously low hardness therein) while no such porosity could be found in other systems. Only the vacancy mechanism of diffusion appears to be useful in explaining these phenomena, though other theoretical points remain to be explored. Inasmuch as the amount of shift is independent of the nature of the external surface of the diffusion couple, and of the dimensions of the couple, it appears that operating vacancles cannot come from external surfaces but must be generated within the diffusion zone itself.

\title{
The Application of Radioactive Tracers to the Study of Metal-Solution Reactions
}

\author{
by M. T. Simnad
}

\begin{abstract}
A $\mathbf{S}$ part of a general project to study the propA erties of metal surfaces, experiments are being carried out designed to elucidate the influence of surface treatment and structure upon the reactions taking place when metals are placed in ionic solutions containing radioactive ions. The behavior of metals in solutions may be studied in greater detall with the ald of radioactive tracers than has been possible with the methods previously available. This applies especially to cases where some of the ions in solution are composed of the same element as the metal, and the tracer technique is then the only method that can be used to follow the reaction.

The first part of the work deals with the socalled phenomenon of "exchange" between metals and radioactive ions in solution. This was flrst demonstrated in 1929 by Hevesy who found that when a specimen of lead was placed in a solution containing thorium $B$ ions it acquired a surface activity corresponding to over 1000 atoms thick of thorium B. No further work appears to have been done on the subject until recent years when Rollin, Coffin and Tingley, and Hensley et al published notes on some experiments confirming this phenomenon with radioactive silver and sodium ions. In our studies, radioactive ions of cobalt, chromium, nickel and iron are being used in solutions into which are immersed numerous metals and alloys with various surface treatments. The effect of the presence and absence
\end{abstract}

M. T. Simnad is associated with the Metals Research $\mathrm{Lab}$ at Carnegie Institute of Technology, Pittsburgh,
$\mathrm{Pa}$. of oxygen in the solutions and of oxide films on the metals, the influence of time, temperature and $\mathrm{pH}$ are being studied. The distribution of the radioactive ions deposited on the metal surfaces is shown by means of autoradiographs and their quantity measured by counting with a Geiger-Müller counter.

The experiments with radioactive cobalt and chromium indicate that the phenomenon of exchange is actually a local electrolysis resulting from differences in electrode potential on the metal surfaces. The experiments with radioactive chromium, in which active chromate ions were used in the solutions, have served to show that passivity in chromate solutions results from the deposition of chromate ions at anodic points on the metal surface, and the distribution of these points following various passivating treatments is brought out in autoradiographs of the specimens. Further experiments have been designed or are in progress using radioactive chromium to study the nature and composition of oxide films on stainless steels and their behavior in solutions, and to throw further light on the phenomenon of grain-boundary sensitization of stainless steels.

In conjunction with these experiments, work is in progress to study the kinetics and mechanism of electrodeposition in very dilute solutions and in its earliest stages. The influence of the numerous factors which affect the nature of electrodeposits will be studied, especially the influence of surface structure upon the nucleation and growth of the deposits. The use of tracers is a highly sensitive tool for the study of this subject. 